\title{
Comparative Study of Conductivity based Silver Modified ZSM5 \& Beta Zeolites as a LPG and $\mathrm{CO}_{2}$ Gas Sensor
}

\author{
A. A. Joshi ${ }^{1}$, R. R. Attarde ${ }^{2}$ \\ ${ }^{1}$ Department of Physics, ACS College, Dharangaon Dist. Jalgaon - 425105 (MS) India \\ ${ }^{2}$ Department of Physics, M.J.College, Jalgaon Dist. Jalgaon-425001(MS) India
}

\begin{abstract}
Zeolites are the gas sensing materials. The microspores of H-Zeolite Socony Mobil-Five (H-ZSM5) and H-Beta zeolites are modified with metal Silver Nitrate $\left(\mathrm{AgNO}_{3}\right)$ by ion exchange method. The modified Silver- ZSM5 (Ag-ZSM5) and Silver-Beta (Ag-Beta) are characterized by X-Ray Diffraction (XRD), Infrared (IR) Spectroscopy, Thermo Gravimetric Analysis (TGA), Differential Thermal Analysis(DTA), Scanning Electron Microscopy (SEM), Energy Dispersive Analysis of X-Ray (EDAX) techniques. Gas sensing characteristics of Ag-ZSM5 and Ag-Beta zeolite have been studied for Liquified Petroleum Gas (LPG) gas and Carbon dioxide (CO $\left.{ }_{2}\right)$ gas. The gas sensing properties of Ag-ZSM5 and Ag-Beta zeolite are studied in a static gas characterization system. Adsorption of gas molecules in zeolite changes the ionic activity, which interns changes conductivity. The maximum Percent Sensitivity Factor (PSF) is measured as a function of operating temperature and as a function of gas concentration for $\mathrm{LPG}_{\mathrm{P} d \mathrm{CO}}$ gas.
\end{abstract}

Keywords: Zeolite, Ion exchange, LPG, EDAX, SEM, PSF

\section{Introduction}

Zeolites are crystalline micro porous aluminosilicates, composed of corner-sharing $\mathrm{AlO}_{4}$ and $\mathrm{SiO}_{4}$ tetrahedra joined into three dimensional frameworks having pores of molecular dimensions. They are a subset of the larger class of molecular sieves. The porous structure consists of cavities, channel and water; also, the cations that counterbalance the negative ions of aluminum sites usually occupy the pore space $[1,2]$.The presence of aluminum in the silica alumina zeolite framework give rise to anion. These anions attract cations and hence, in the framework cations are present and they are loosely bound with the framework. The replacement of cation held in the framework structure is possible by the other cation. Hence ion exchange is possible. [3] Since cations are free, they can move to new positions under the influence of an external electric field, which causes the electric charge transport. It is conductivity and dielectric relaxation.

Zeolite possesses classical applications like adsorption [4], catalyst $[5,6]$, and the possibility of zeolite pores to host different ions, atoms, molecules and clusters have opened up numerous opportunities. In last few years, zeolites have been used in the field of novel technologies such as chemical sensors [7, 8, and 9]. As a sensing material, zeolites are very favourable due to its additional high thermal stability and chemical resistance. Zeolite possesses a remarkable property of hydration and dehydration on heating without any major changes in structure. Adsorption of gas molecules in zeolite micropores changes the ionic activity and hence conductivity changes. This property is used as a gas sensor in the present study.

Propane $\left(\mathrm{C}_{3} \mathrm{H}_{8}\right)$ is the hazardous gas to be detected due to its flammability. It is a green house gas to be detected and also one of the major constituents of LPG gas. Carbon dioxide $\left(\mathrm{CO}_{2}\right)$ is a non -toxic gas, it is one of the important gas to monitor with respect to "Green House Effect". $\mathrm{CO}_{2}$ is a good indicator of air quality pollution. So the detection and measurement of presence of $\mathrm{CO}_{2}$ and LPG gas concentration in the atmospheres of offices and houses are necessary. In the present study zeolite material is used as sensor to sense presence these gases.

\section{Materials and Methods}

In the present study, the gas sensing properties of SilverZSM5 (Ag-ZSM5) and silver-Beta (Ag-Beta) zeolites are studied for $\mathrm{LPG}$ and $\mathrm{CO}_{2}$ gas.

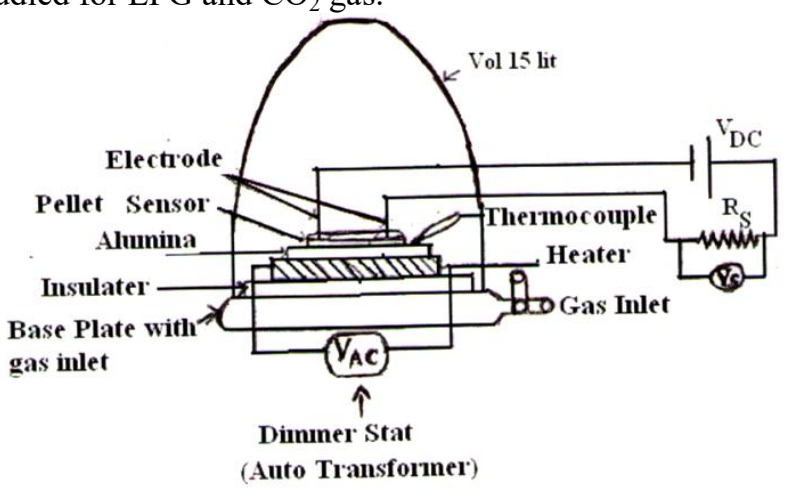

Figure 1 (a): Experimental set of gas sensor

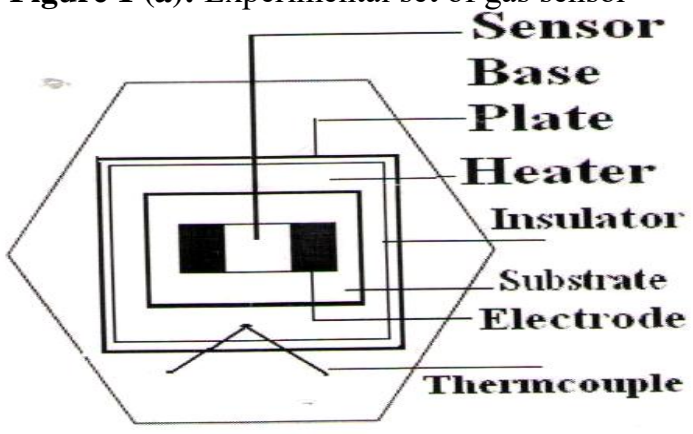

Sensor Assembly

Figure 1 (b): Sensor Assembly 


\section{International Journal of Science and Research (IJSR) \\ ISSN (Online): 2319-7064}

Index Copernicus Value (2013): 6.14 | Impact Factor (2015): 6.391

Static Gas Characterization System is used to study gas sensing property which is shown in figure 1(a). A figure 1(b) gives the details of sensor assembly [10]. The system consist of heater of nichrome wire $(1.5 \mathrm{~kW}$ and $\mathrm{R}=120 \Omega)$. It is used to vary the substrate temperature from $345 \mathrm{~K}$ to $575 \mathrm{~K}$ with the help of dimmer stat. Temperature of the sample is measured with the help of temperature indicator with $\mathrm{Cr}-\mathrm{Al}$ thermocouple. The electrical terminals are brought out from the sensor assembly by using insulated bead- through mounted on the stainless steel base plate. The base plate has a gas inlet and on which a glass chamber (Volume $\approx 15$ lit) is mounted. The spring press contact electrodes are used during the measurements of gas sensing properties. Half bridge method is used to determine the conductance of the zeolite sample. The resistance of the sample is measured directly by electrometer (Keithely Multi Meter). The required gas concentration inside the system is achieved by injecting a known volume of the gas in the airtight chamber at ambient conditions. The DC conductance of the sample is obtained by applying the voltage $(\mathrm{V})$ through variable DC power supply $(0-250 \mathrm{~V})$ to sensor and measuring the voltage drop Vs across Rs. The conductance $\mathrm{G}$ and the percentage sensitivity factor $(\% \mathrm{SF})$ are calculated by using the following formula.

$$
\begin{gathered}
G=\frac{V_{s}}{\left[R_{s}\left(V-V_{s}\right)\right.} \\
\% S . F .=\left[\frac{\left(G_{g}-G_{a}\right.}{G_{a}}\right] \times 100=\left(\frac{\Delta G}{G_{a}}\right) \times 100
\end{gathered}
$$

Where, $G_{a}$ and $G_{g}$ are the conductance of the sensor in air and gas + air respectively.

\section{Experimental}

Sensor materials were prepared by the synthesized ZSM5[11] and Beta [12] zeolite. The ZSM-5 and Beta was modified with $4 \mathrm{~g}$ of Silver Nitrate $\left(\mathrm{AgNO}_{3}\right)$. Silver Nitrate was taken in $250 \mathrm{ml}$ round bottom flask and $30 \mathrm{ml}$ methanol and $10 \mathrm{gm}$ of dried ZSM-5 zeolite was added. The mixture was stirred using magnetic stirrer for 15 minute for homogenization and placed under reflux condition at $338 \mathrm{~K}$ for $12 \mathrm{hrs}$. Further, it was washed repeatedly after reaching to room temperature. Then it is dried in an oven at $383 \mathrm{~K}$ for overnight. The modified ZSM5 is characterized by XRD, IR, TGA-DTA, SEM and EDAX. The modified silver ZSM5 (Ag-ZSM5) was mixed with polyvinyl alcohol (PVA) as a binder material and is pressed to form pellet (diameter $13 \mathrm{~mm}$ and thickness $1 \mathrm{~mm}$ ) using hydraulic press. The pellet is then fired at $573 \mathrm{~K}$ for $4 \mathrm{hrs}$ to drive out the binder material (PVA). Silver paste is coated on the surfaces of the pellet to ensure good electrical contacts. The similar procedure was adopted for the preparation of modified form of Silver-Beta (Ag-Beta) zeolite. For the preparation of AgBeta pellet same procedure, as mentioned above, is done. The ion exchanged sample of Ag-Beta is characterized using XRD, IR, TGA-DTA, SEM and EDAX. X-Ray diffractogram (XRD) of the zeolite samples were taken using $\mathrm{CuK}_{\alpha}$ radiation at a scanning speed of 1.2 degree per $\mathrm{min}$. The zeolite material is scanned in the $2 \theta$ range of $20^{\circ}$ to $80^{\circ}$. The XRD pattern was obtained at $30 \mathrm{kV}$ and $15 \mathrm{~mA}$ on Philips (3710pw/1710) system.
The XRD of synthesized ZSM5 and Beta samples are compared with standard data and are in agreement. The XRD of ion-exchanged samples are similar to that of original samples, indicating that there is no change in crystal structure. The XRD pattern of Ag-ZSM5 zeolite is shown in fig. 2 and of Ag-Beta zeolite in fig.3.

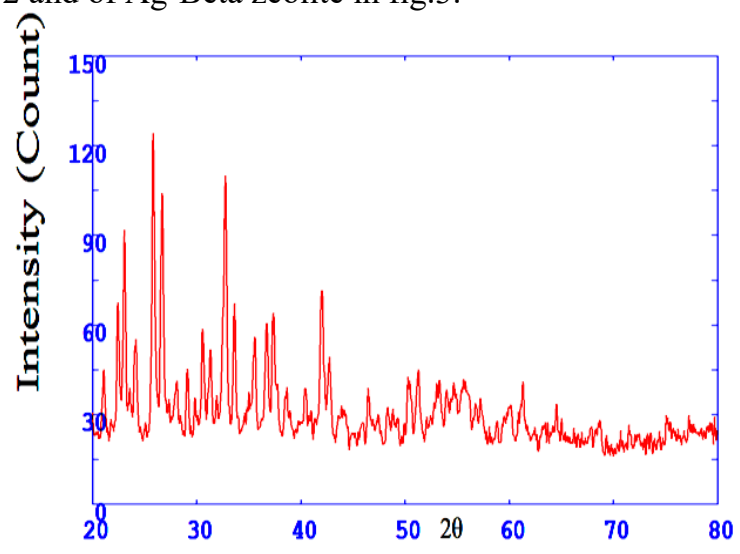

Figure 2: XRD pattern for Ag-ZSM5 Zeolite

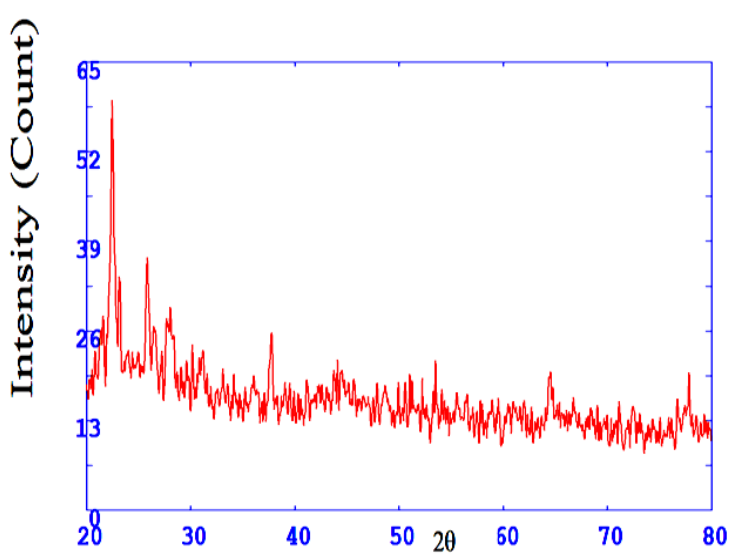

Figure 3: XRD pattern for Ag-Beta Zeolite

Infrared (IR) Spectra of Ag-ZSM5 and Ag-Beta zeolite is recorded on Perkin-Elmer FT-IR spectrophotometer in the frequency range $450-4000 \mathrm{~cm}^{-1}$ by using $\mathrm{KBr}$ pellet technique. This is shown in fig.4 for Ag-ZSM5 zeolite and in fig. 5 for Ag-Beta zeolite.

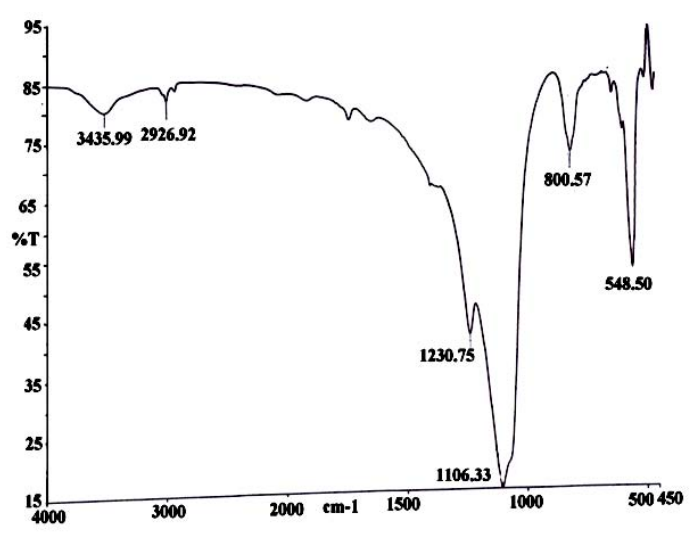

Figure 4: IR Spectra of Ag-ZSM5 zeolite 


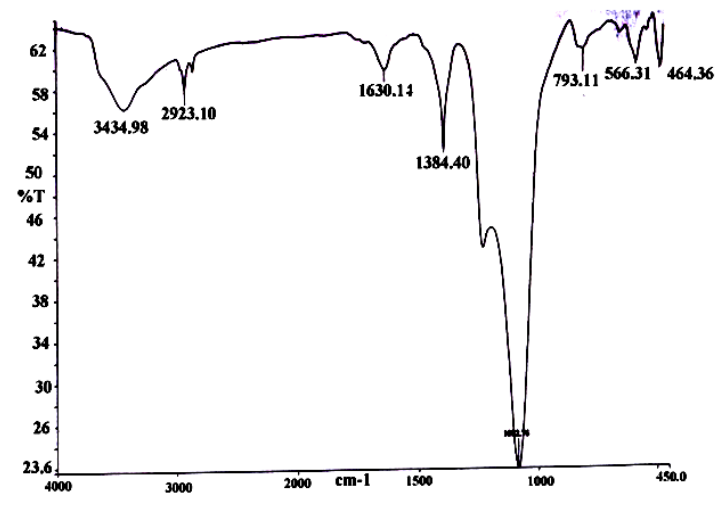

Figure 5: IR Spectra of Ag-Beta zeolite

In Ag-ZSM5 zeolite the structure insensitive asymmetric stretch and symmetric stretch bands are found to be at 1106 $\mathrm{cm}^{-1}$ and $800 \mathrm{~cm}^{-1}$ respectively. The T-O bend is observed at $460 \mathrm{~cm}^{-1}$. The structure sensitive asymmetric stretch band is observed at $1230 \mathrm{~cm}^{-1}$. The band near $548 \mathrm{~cm}^{-1}$ is the characteristic of the presence of double five member ring [14]. The water bands are present at $3435.9 \mathrm{~cm}^{-1}$ and 1650 $\mathrm{cm}^{-1}[15]$.

In Ag-Beta zeolite structure insensitive asymmetric stretch band is observed at $1082.7 \mathrm{~cm}^{-1}$ and symmetric stretch band is at $793 \mathrm{~cm}^{-1}$. The T-O bend is found to be at $464 \mathrm{~cm}^{-1}$. The structure sensitive asymmetric stretch band found at 1230 $\mathrm{cm}^{-1}$. The double ring which is the characteristics of highly crystalline Beta zeolite is observed at $566 \mathrm{~cm}^{-1}$. The water bands are observed at $3434 \mathrm{~cm}^{-1}$ and $1630 \mathrm{~cm}^{-1}$. These bands are the characteristics of zeolite material.

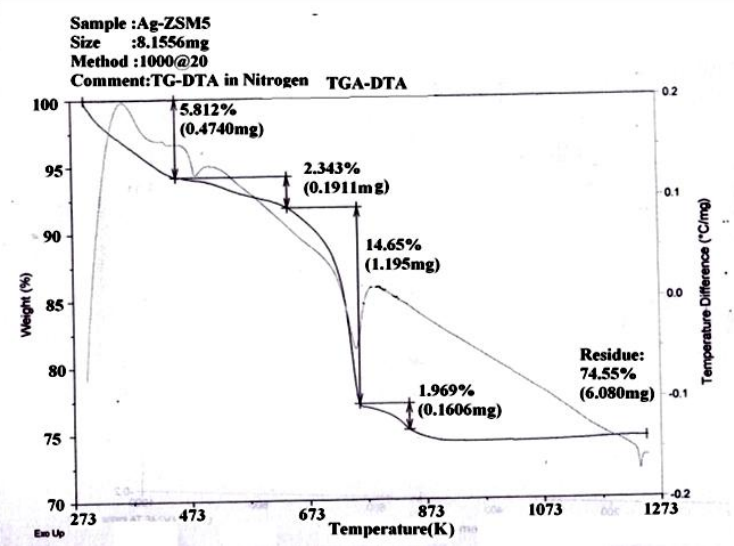

Figure 6: TGA-DTA curve of Ag-ZSM5 Zeolite

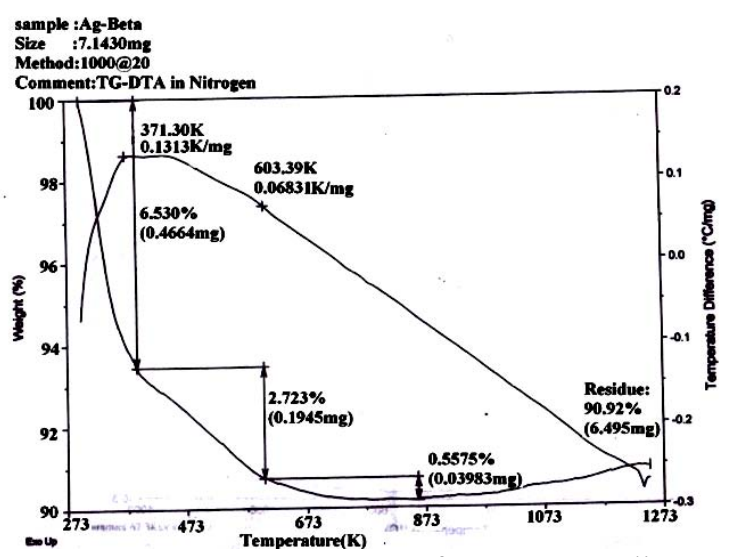

Figure 7: TGA-DTA Curve of Ag-Beta Zeolite
The TGA-DTA spectra were studied on T.A. instrument (U.S.A.) SDT-2960 with reference material $\mathrm{Al}_{2} \mathrm{O}_{3}$ in nitrogen atmosphere. The TG/DTA curves for Ag-ZSM5 and Ag-Beta zeolite are shown in fig. 6 and fig.7. A TGA curve for Ag-ZSM5 shows four steps of weight loss with total weight loss of $25.45 \%$. In first step the weight loss is of $5.812 \%$, which is in the temperature range of $300 \mathrm{~K}$ to $450 \mathrm{~K}$, and is due to the loss of molecular and adsorbed water [16]. The second step in the temperature range of $450 \mathrm{~K}$ to $650 \mathrm{~K}$, the weight loss of $2.343 \%$, which corresponds to removal of Tetra Ethyl Ammonium Bromide (TEA-Br) and which was used as a structure direacting agent while synthesizing ZSM5 zeolite. The weight loss is highest in third step and it is $14.6 \%$, in the temperature range of $650 \mathrm{~K}$ to $750 \mathrm{~K}$ [17]. This weight loss is due to oxidative decomposition of templates and water loss [18]. The weight loss of $1.9 \%$ is observed in the temperature range $750 \mathrm{~K}$ to $850 \mathrm{~K}$. It is due to dehydration of sorbed water inside the super cages. Above $850 \mathrm{~K}$ no weight loss is observed.

The DTA analysis shows two endotherms. These are observed in Ag-ZSM5 zeolite at $437 \mathrm{~K}$ and at $750 \mathrm{~K}$ temperature. This indicates that the immobile water in pores is removed at $437 \mathrm{~K}$ and the physical change is taking place at $750 \mathrm{~K}$ [19]

Ag-Beta zeolite is potentially the most hydrophilic, was subjected to TG analysis to evaluate the water content [20]. The weight loss is observed in three steps in Ag-Beta zeolite. In first step the weight loss is maximum $6.53 \%$ in the temperature range $300 \mathrm{~K}$ to $400 \mathrm{~K}$. This is due dehydration of physically sorbed water in Ag-Beta zeolite cavities [19]. The weight loss is $2.723 \%$ is observed in second step in the temperature range of $400 \mathrm{~K}$ to $600 \mathrm{~K}$. This weight loss seems to be due to decomposition of (Tetra Ethyl Ammonium) TEA + ions and mobile water coming out of zeolite cavities [21]. The weight loss of $0.55 \%$ is observed in third step, when is in the temperature range $600 \mathrm{~K}$ to $873 \mathrm{~K}$. This is due to decomposition of TEA-Br, a structure directing agent which was used during synthesis. Above $873 \mathrm{~K}$ no weight loss is observed. In DTA analysis shows, no endotherm is observed in Ag-Beta zeolite, indicating no structural change up to $1200 \mathrm{~K}$.

The morphology of the zeolites was investigated using scanning electron microscope, Leo-Leica, Sterioscan model 440, Cambridge (U.K.). Figure 8 shows the microgram of Ag-ZSM5 zeolite, wherein cubic and needle shaped zeolite crystals are observed. These crystals are $3 \mu \mathrm{m}$ in size. Figure 9 shows the microgram of Ag-Beta zeolite. The crystallites are spheroid shaped having crystal size of $3 \mu \mathrm{m}$. 


\section{International Journal of Science and Research (IJSR) \\ ISSN (Online): 2319-7064}

Index Copernicus Value (2013): 6.14 | Impact Factor (2015): 6.391

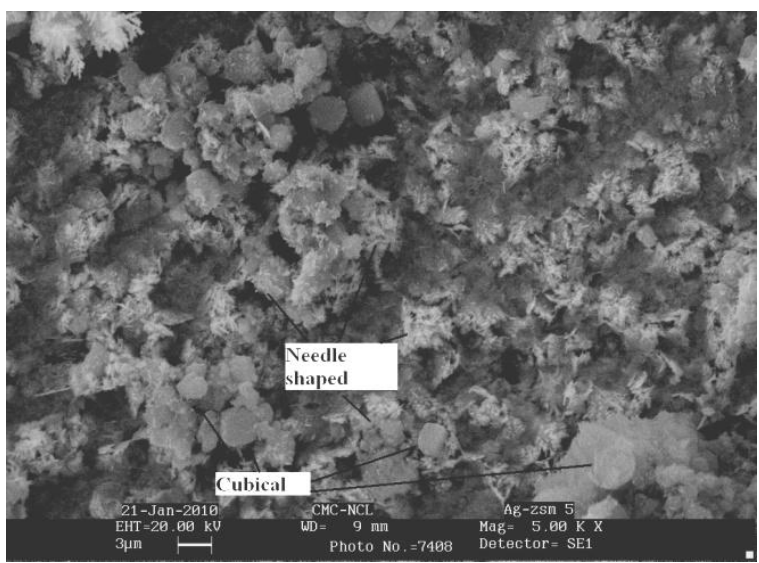

Figure 8: SEM photo graph of Ag-ZSM5

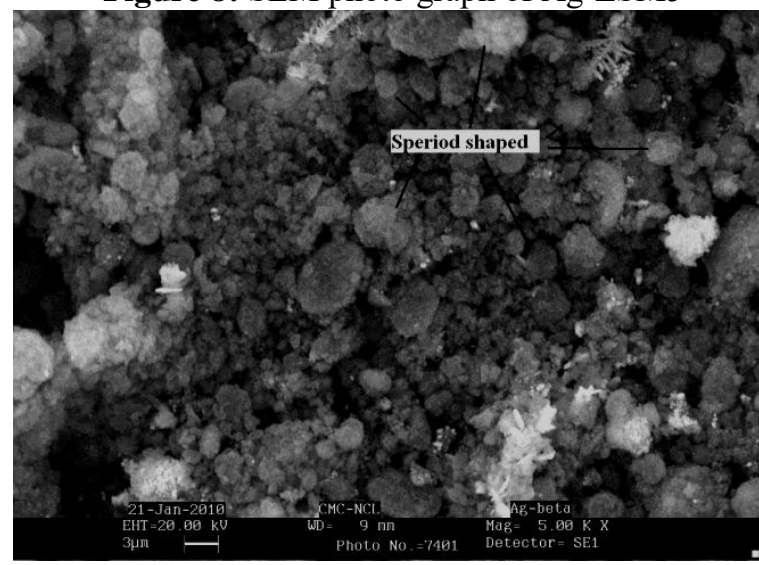

Figure 9: SEM photo graph of Ag-Beta

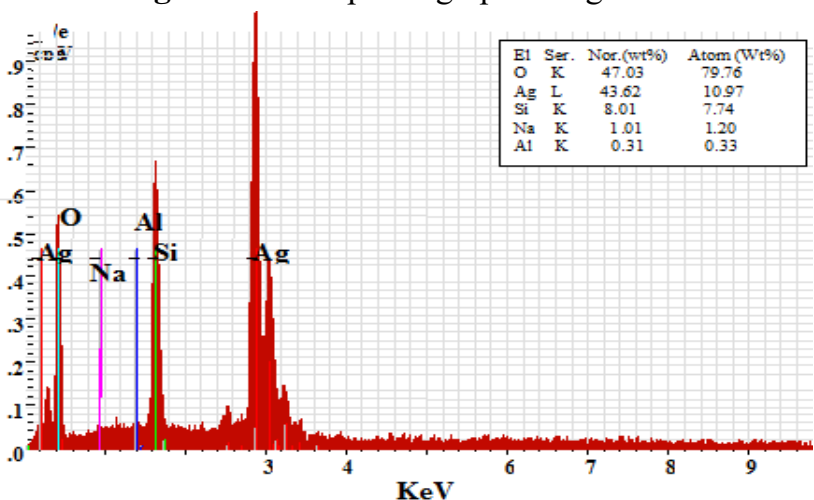

Figure 10: EDAX of Ag-ZSM5

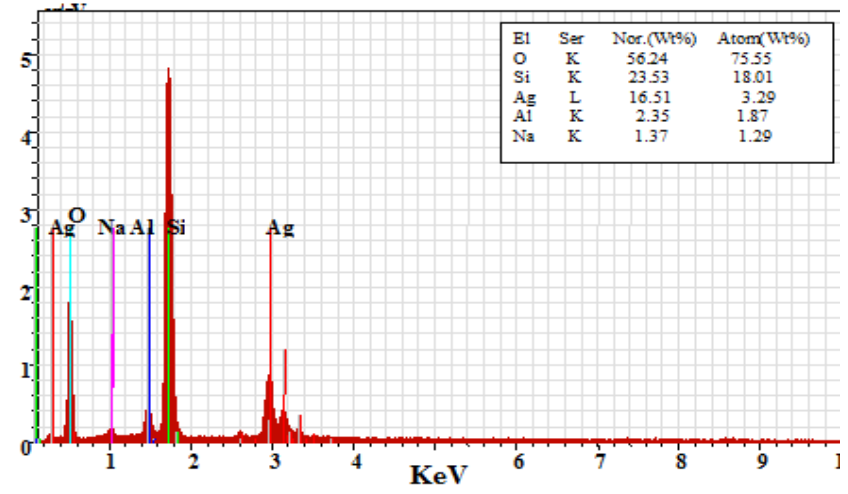

Figure 11: EDAX of Ag-Beta

The elemental analysis is done with the help of EDAX; (Model No. Quanta X-200, Bruker, Germany). The EDAX analysis of Ag-ZSM5 is shown in fig. 10 and of Ag-Beta in fig. 11. The elemental analysis of Ag-ZSM5 and Ag-Beta is given in figure. The elemental analysis indicates that the silver ions are incorporated in Ag-ZSM5 and Ag-Beta zeolite. The $\mathrm{Si} / \mathrm{Al}$ ration of $\mathrm{Ag}-\mathrm{ZSM} 5$ and $\mathrm{Ag}$-Beta is found to be 23 and 9.6 respectively.

\section{Result and Discussion}

Fig. 12 shows the typical variation of the \%sensitivity as a function of operating temperature at $1000 \mathrm{ppm}$ for LPG gas for Ag-ZSM5 and Ag-Beta zeolite. The maximum PSF for Ag-ZSM5 zeolite is found to be $49 \%$ at temperature $568 \mathrm{~K}$ for LPG. Other peaks have been observed at $368 \mathrm{~K}, 433 \mathrm{~K}$, $468 \mathrm{~K}$ but the percent sensitivity factor is less than $49 \%$ (Table1). Several studies have been reported on changes of the complex impedance of zeolite when exposed to hydrocarbons [21, 22]. It was found that conductivity of sensing material increases with increasing hydrocarbon contents [23].Marginal higher temperatures enhance ion mobility in the zeolite lattice leading to a marginal higher value of conductivity. In present study, it is the maximum PSF $(71 \%)$ at $388 \mathrm{~K}$ is observed for Ag-Beta zeolite for LPG gas. The pore size of ZSM5 zeolite $5.4 * 5.6 \AA$ and Beta zeolite is $7.6 * 6.4 \AA$. The molecular diameter of LPG (Propane and Butane) is $4.3 \AA$. Beta zeolite may be adsorbing more LPG molecules as compare to ZSM5 zeolite. It seems that Ag-Beta zeolite is more suitable material for sensing LPG gas at fairly low temperature.

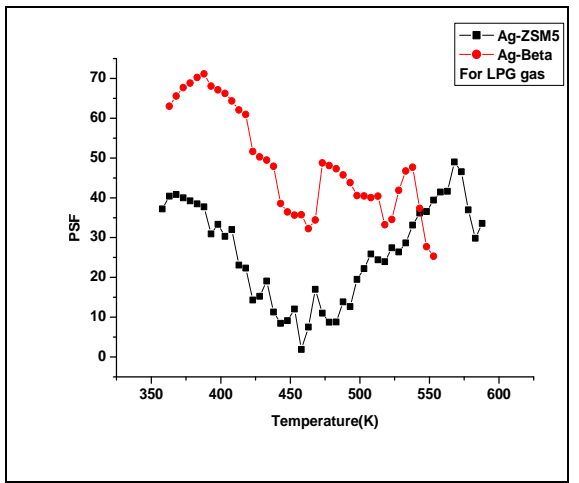

Figure 12: \% S. F. as a function of temperature for LPG.

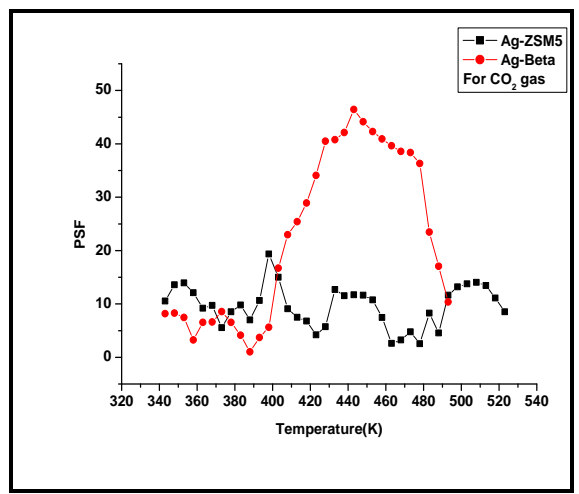

Figure 13: $\%$ S. F. as a function of temperature for $\mathrm{CO}_{2}$ gas

Table 1: Sensor parameter of LPG

\begin{tabular}{|c|c|c|}
\hline Parameters & \multicolumn{2}{|c|}{ Samples } \\
\hline & Ag-ZSM5 (568K) & Ag-Beta (388K) \\
\hline \% S.F. & $49 \%$ & $71 \%$ \\
\hline $\begin{array}{c}\text { Saturation Point } \\
\text { (Gas Concentration) }\end{array}$ & $12,500 \mathrm{ppm}$ & $\begin{array}{c}\text { No saturation is } \\
\text { observed }\end{array}$ \\
\hline
\end{tabular}




\section{International Journal of Science and Research (IJSR) \\ ISSN (Online): 2319-7064}

Index Copernicus Value (2013): 6.14 | Impact Factor (2015): 6.391

Table 2: Sensor parameter of $\mathrm{CO}_{2}$ gas

\begin{tabular}{|c|c|c|}
\hline Parameters & \multicolumn{2}{|c|}{ Samples } \\
\hline & Ag-ZSM5 (443K) & Ag-Beta (498K) \\
\hline \% S.F. & $49 \%$ & $19 \%$ \\
\hline $\begin{array}{c}\text { Saturation Point } \\
\text { (Gas Concentration) }\end{array}$ & $20,500 \mathrm{ppm}$ & $20,500 \mathrm{ppm}$ \\
\hline
\end{tabular}

Fig. 13 gives presentation of PSF as a function operating temperature for Ag-ZSM5 and Ag-Beta zeolite for $\mathrm{CO}_{2}$ gas. For Ag-ZSM5 zeolite the maximum PSF observed for $\mathrm{CO}_{2}$ gas is $49 \%$ at temperature $443 \mathrm{~K}$. PSF observed for Ag-Beta zeolite for $\mathrm{CO}_{2}$ gas is $13.5 \%, 19 \%, 13 \%$ at temperature $348 \mathrm{~K}, 398 \mathrm{~K}$, and $433 \mathrm{~K}$ respectively. The maximum PSF $19 \%$ is observed at $398 \mathrm{~K}$ temperature (Table2). The sensitivity observed may be due to chemical reaction occurring between $\mathrm{Ag}-\mathrm{CO}_{2}$ [24] and thereby an acceptance or release of an electron is possible to produce a change in the conductivity of the zeolite. The variation of PSF as a function of operating temperature study reveals that Ag-Beta zeolite is found to be more sensitive for LPG gas compare to $\mathrm{CO}_{2}$ gas.

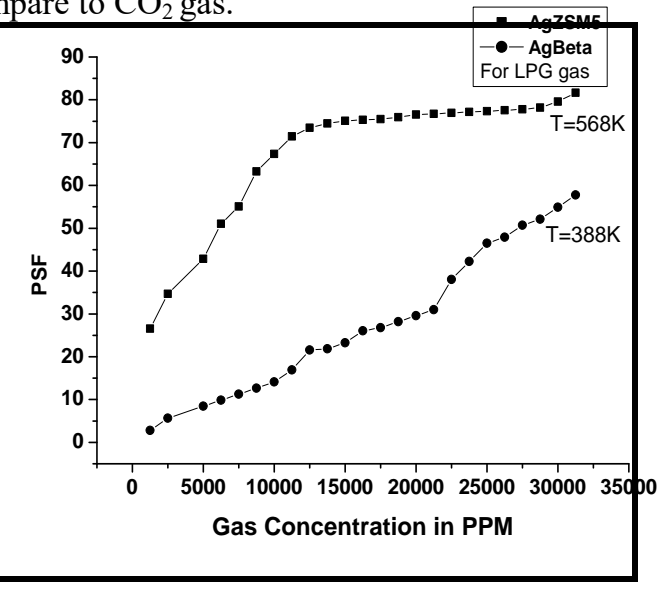

Figure 14: \% S. F. as a function of concentration for LPG

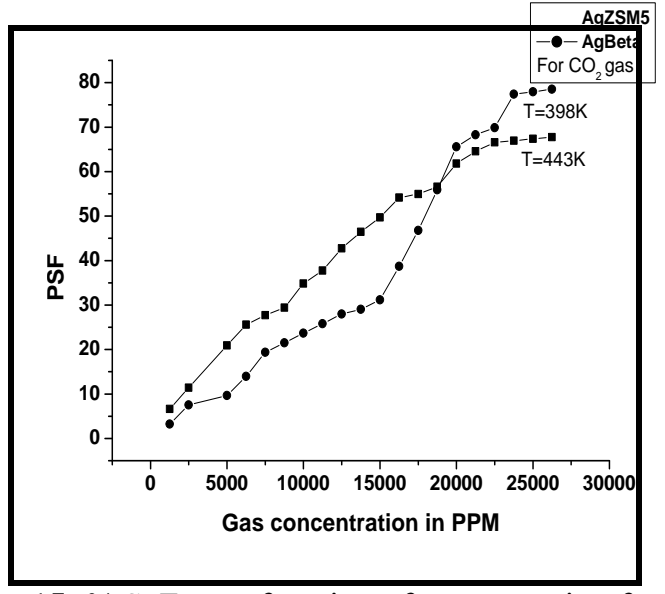

Figure 15: $\%$ S. F. as a function of concentration for $\mathrm{CO}_{2}$

Fig.14 shows variation of PSF as a function of gas concentration of Ag-ZSM5 and Ag-Beta zeolites at temperatures $568 \mathrm{~K}$ and at $388 \mathrm{~K}$ respectively for LPG gas. In Ag-ZSM5 the PSF increases with increasing gas concentration up to $12500 \mathrm{ppm}$ remains constant thereafter. For Ag-Beta PSF as a function of gas concentration is quasi linear. The PSF increases from $1250 \mathrm{ppm}$ to $22500 \mathrm{ppm}$ linearly and again from 22500ppm to $32500 \mathrm{ppm}$ steeply. $\mathrm{PSF}$ as a function of gas concentration for $\mathrm{CO}_{2}$ gas for $\mathrm{Ag}-$ ZSM5 and AG-Beta is shown in fig. 15. The PSF increases with gas concentration up to 20500ppm and remains constant, thereafter for Ag-ZSM5 zeolite. The PSF increases from $1250 \mathrm{ppm}$ to $15000 \mathrm{ppm}$ slowly and there is rapid increase in PSF between15000ppm to 20500ppm. Above 20500ppm the PSF remains almost constant for Ag-Beta zeolite.

\section{Conclusions}

PSF as a function of temperature for LPG gas: Ag-Beta zeolite senses the LPG gas at fairly low temperature as compared to Ag-ZSM5 zeolite. The PSF is $71 \%$ at $388 \mathrm{~K}$ for Ag-Beta zeolite. Hence, Ag-Beta zeolite is more suitable for LPG gas.

PSF as a function of gas concentration for LPG gas: AgZSM5 saturates at $12,500 \mathrm{ppm}$ gas concentration, whereas such a saturation point is not observed in Ag-Beta zeolite. Hence, in this case no definite conclusion can be drawn.

PSF as a function of temperature for $\mathrm{CO}_{2}$ gas: Ag-ZSM5 senses the $\mathrm{CO}_{2}$ gas at $443 \mathrm{~K}$ with 49PSF. Ag-Beta senses the $\mathrm{CO}_{2}$ gas at $498 \mathrm{~K}$ with 19PSF. Hence, Ag-ZSM5 zeolite is suitable to sense the $\mathrm{CO}_{2}$ gas.

PSF as a function of gas concentration for $\mathrm{CO}_{2}$ gas: As a function of gas concentration Ag-ZSM5 and Ag-Beta saturates at 20,500ppm. But, Ag-ZSM5 saturates at low temperature. Hence, it is more suitable to sense the $\mathrm{CO}_{2}$ gas.

\section{References}

[1] Baerlocher C.H., Meier W.H., Olson D.H., Atlas of Zeolite Framework Type, $5^{\text {th }}$ ed, Elsevier , Amsterdam, (2001)

[2] Mc Cucker L.B., Liebau F., and Englhardtal G., Pure. Appl. Chem. 73(2001)1.

[3] Break D.W., Zeolite Molecular Sieves, Chemistry and Use (John Wiley and Sons, Inc. New York) chapter 7 and 8, (1974).

[4] Restrepo M.L., Mosquera M.A., Accrete correlation , thermo chemistry, and structural interpretation of equilibrium adsorption isotherms of water vapor in zeolite $3 \mathrm{~A}$ by means of a generalized statistical thermodynamic adsorption model, 283 (2009) 73 - 88.

[5] Corma A, State of art and future challenges of zeolites as catalysis, Journal of catalysis, 216 (2002) 298-312.

[6] Marcilla A., Gomez-Siurana A., Valdes F., Catalysis pyrolysis of LDPE over H-Beta and H- ZSM5 zeolite in dynamic conditions: Study of the evolution of the process, Journal of Analytical And Applied Pyrolysis, Volume 79 (2007) 433-442.

[7] Hagen G., Schulz A., Kmber M., Moos R., Four-Wire Impedance Spectroscopy on Planar Zeolite/Chromium Oxide Based Hydrocarbon Gas Sensors, Sensors 7 (2007) 2681 - 2692.

[8] Reib S., Hagan G., Moos R., Zeolite-based Impedimetric Gas Sensor Device in Low-cost Technology for Hydrocarbon Gas Detection, sensors, 8 (2008) 7904 - 7916.

[9] Sahner K.,Hagen G., Schönauer D. Reiß S. and Moos R. Zeolites, Versatile materials for gas sensors, Solid State Ionics, 179 (2008) 2416 - 2423.

\section{Volume 5 Issue 4, April 2016}


[10] Joshi S.K., Rao C.N.R., (New materials, Narosa publishing House, T.Tsuruto, S. Nagakura, New Delhi) (1992) 1

[11] Somani O.G., Choudhari A.L., Rao B.S., Mirajkar S.P., Material Chemistry and Physics, 82 (2003) 538 545.

[12] Camblor M.A., Mifsued A., Rarientz J.P., Crystallization of zeolite Beta: Effect of $\mathrm{Na}$ and $\mathrm{K}$ ions, Zeolites, 1(1991) 202.

[13] Zhdanov S.P., Kosheleva L.S., Titova T.I. and Shubaeva M.A., Effect of alkaline modification of products of thermal vacuum and hydrothermal treatment of $\mathrm{NH}_{4} \mathrm{Na}-\mathrm{Y}$ zeolite according to the IR spectral data, Russian Chemical Bulletin,41 (1992) $1341-1345$.

[14] Flanigen E.M., Khatami H., and Szymanski H.A., in Adv. Chem.Ser.,Flanigaen E.M., Sand L.B.(eds.) American Society, Washington D.C.101 (1971) 201 228.

[15] Jacobs P.A., Beyer H.K., Alyon J.V., Properties of the end members in the pentasil-family of zeolites: Characterization as adsorbents, zeolites 1 (1981) 161.

[16] Mohamed R.M., A li M.H., El-Shahat M.H., Ibrahim I.A., Effect of the silica sources on the crystallinity of nanosized ZSM-5 zeolite, Microporous and Mesoporous 79 (2005) 7 - 12.

[17] Larlus O., Valtchev V., Synthesis of all-silica BEAtype material in basic media, Microporous and Mesoporous, 93 (2006) 55 - 61.

[18] Joshi M.S., Joshi U.V., Choudhari A.L., M.W. Kasture, Structural studies of natural heulandites using infrared spectroscopy, Material Chemistry and Physics, 48 (1997) 160 - 163.

[19] Kasture M.W., Joshi V.V., Choudhari A.L., Thermal Properties of ion exchanged natural stalbites, Research Journal of S.R.T. Marathwada University Nanded, (1999) 13 - 20.

[20] Camblor M.A., Corma A., and Valencia S., Synthesis in fluoride media and characterization of aluminosilicate zeolite Beta, Journal of Materials chemistry, 8 (1998) 2137 - 2145.

[21] Alberti K., Hanns J., Plog C., Fetting F., Zeolite Coated inter digital capacitors as a new type of gas sensor, Catal. Today 8 (1991) 509-513.

[22] Alberti K., Gubicza L., Fetting F., Gas sensoren ftir Kohlenwasserstoffe mit katalytisch aktiven YZeolithen (Hydrocarbon gas sensors utilizing catalytic active zeolite Y), Chem. Ing. Tech. 65 (1993) 940 943.

[23] Alberti K., Fetting F., Zeolites as sensitive materials for dielectric gas sensors, Sensors and Actutators B Chem, 21 (1994) 39-50.

[24] Hagen G., Dubbe A., Rettig F., Jerger A., Birkhofer Th., Muller R. ,Plog C. , Moos R., Selective impedance based gas sensors for hydrocarbons using ZSM-5 zeolite films with chromium(III) oxide interface, Sensors and Actuators B 119 (2006) 441448. 